\title{
QUASI-LIKELIHOOD AND ITS APPLICATION by Christopher C. Heyde
}

\author{
A BOOK REVIEW \\ JORDAN STOYANOV \\ Universidade Federal do Rio de Janeiro \\ Departamento de Estatistica, Instituto de Matematica \\ Rio de Janeiro 21945-970, Brazil \\ E-mail jordan@dme.ufrj.br
}

(Received June, 1998)

The new abbreviation QLE will become quite soon as common as are, e.g., the classical MLE and LSE. There are several and serious reasons for that. If you want to know why, you can read this review but do not miss the chance to read the book under review.

There are non-standard and non-trivial but important statistical inference problems for probability distributions or for stochastic processes, which are not tractable by using the classical methods such as MLE or LSE. Due to works of V.P. Godambe, J. Durbin, D.L. McLeish, C.G. Small, and, especially, of the author of the present book, a new methodology was suggested, developing both the general theory and a large number of specific stochastic models. The name "Quasi-Likelihood Estimation" (QLE) came to life, several interesting problems were successfully solved, and for an increasing number of stochasticians QLE became the main field of interests.

Let us mention that the QL approach can be applied to any stochastic model whose description involves only conditions on the first two moments, or more precisely, on the mean and the covariance structure of the model under study. The basic idea of the QLE is to work with appropriate functions called estimating functions. The latter term was suggested by B.F. Kimball (1946), but the idea originates with K. Pearson (1894). The estimating functions depend on both the data and the unknown parameters, so now the estimators come as roots when equating these functions. The estimating functions correspond to the score functions in the MLE. This fact immediately explains why the QL estimation is such a "martingale" in essence. It is not surprising that martingale theory is so strongly involved in QL. First, martingale stochastic models are very natural when describing many phenomena, and second, all the important properties of QL estimators can be derived in a general setting by using the LLN and the CLT for martingales. QL enables one to unify the advantages of the traditional methods of estimation such as ML, LS, conditional LS, minimum chi-squared, and M-estimation. Moreover, this is done under mild regularity conditions. An essential advantage of the QL framework is the opportunity to choose the family of estimating functions to work with. 
While the classical estimation theory deals primarily with independent and identically distributed observations, the QL approach allows one to cover at once a wide spectrum of random dynamical models with both discrete and continuous time.

At the very beginning of the book, the author expresses his point of view that $\mathrm{QL}$ is not simply a method, but rather a methodology; and this reviewer should add: this is a powerful methodology. When reading the book we really understand how deep and effective this approach is when solving diverse inference problems in a very satisfactory way.

New and difficult problems from the contemporary stochastics and its applications are presented with an impressive clarity. Let us look at the names of the chapters:

1. Introduction

2. The General Framework

3. An Alternative Approach: E-Sufficiency

4. Asymptotic Confidence Zones of Minimum Size

5. Asymptotic Quasi-Likelihood

6. Combining Estimating Functions

7. Projected Quasi-Likelihood

8. Bypassing the Likelihood

9. Hypothesis Testing

10. Infinite Dimensional Problems

11. Miscellaneous Applications

12. Consistency and Asymptotic Normality for Estimating Functions

13. Complements and Strategies for Application

References

Index

However, to read the book is much more enjoyable than simply seeing the contents. In a master style the author demonstrates by example how a particular property can be developed into a general result. Both the ideas and techniques are well presented.

The book is written by one of the leading stochasticians of our time. The contributions of Professor Heyde in the field of statistical inference has been very essential. This makes him the most appropriate author of the first book in the field of QL estimation. He did a great job and, therefore, he absolutely deserves the thanks of the stochastics community.

This book can be used very successfully as a text book for graduate courses for students specializing in probability and statistics and their applications. The material is well organized and the fundamental concepts are well explained. All the statements are illustrated by interesting examples and exercises given in details. This reviewer is enthusiastically looking for the nearest possibility to teach such a course to university students based entirely on the book under review, and he believes that such a course will be interesting, useful, and attractive.

This book is not only highly recommended to the libraries, but also to any stochastician dealing with inference problems, both teaching and research, including areas such as industrial applications, population theory, econometrics and financial business.

Surely, QL estimation theory is under an active study; so we can expect new and essential progress in the future. However, this book will remain a cornerstone for the years to come. 
Conclusions about the book: contemporary topics presented in a master style, reasonable size, reasonable price, prospective applications, source for an intellectual pleasure. What more?

Quasi-likelihood and its Application

by Christopher C. Heyde

Publisher Springer-Verlag, New York

Publication Year 1997

ISBN 0-387-98255-6, ix +253 pp.

Price: $\$ 54.95$ 


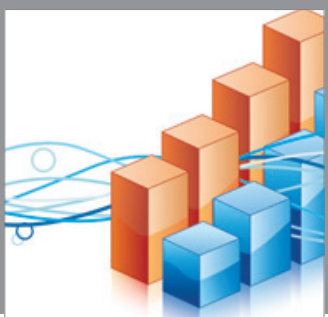

Advances in

Operations Research

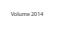

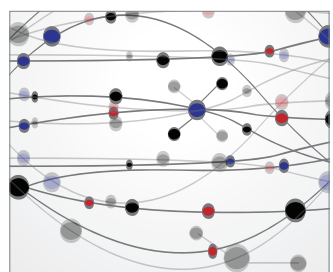

\section{The Scientific} World Journal
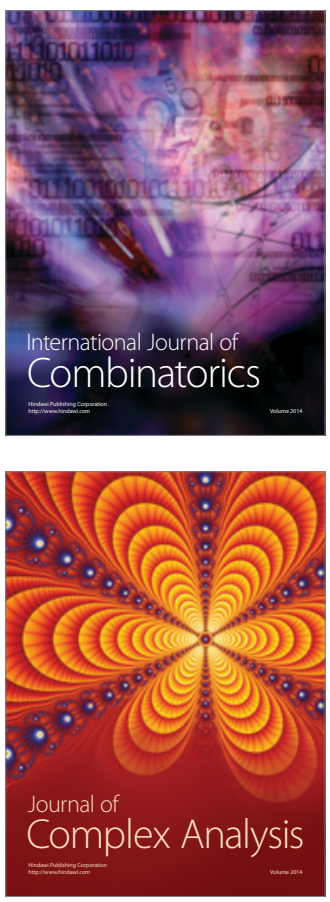

International Journal of

Mathematics and

Mathematical

Sciences
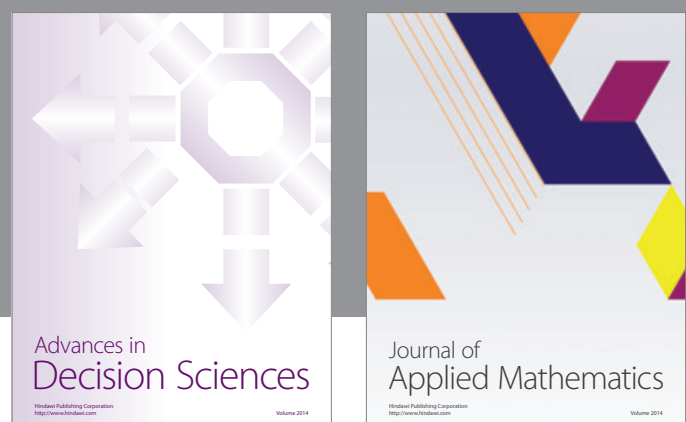

Journal of

Applied Mathematics
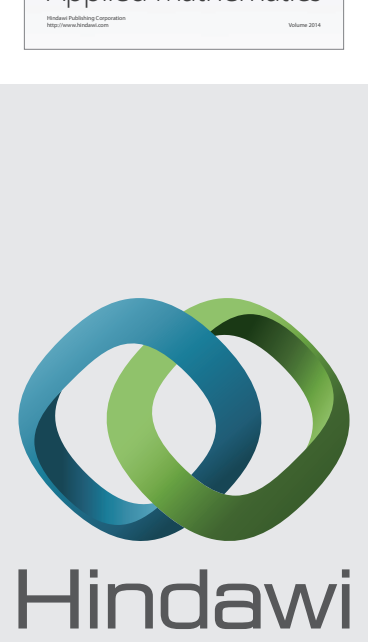

Submit your manuscripts at http://www.hindawi.com
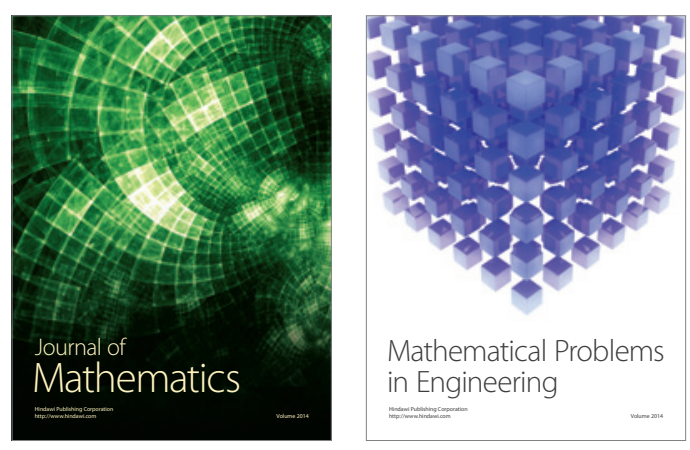

Mathematical Problems in Engineering
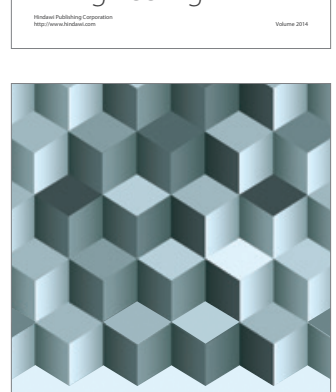

Journal of

Function Spaces
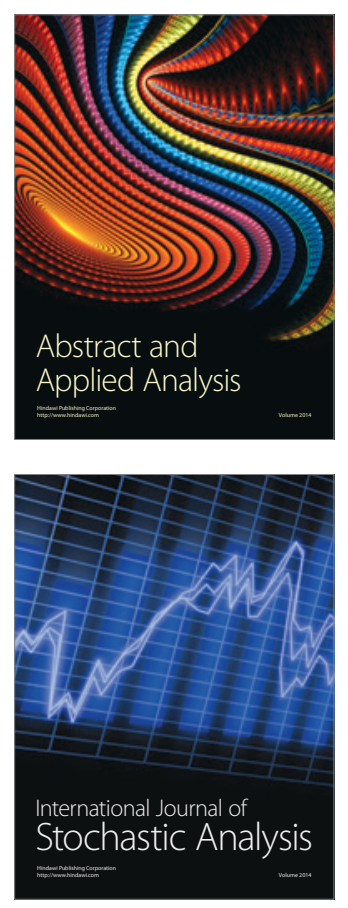

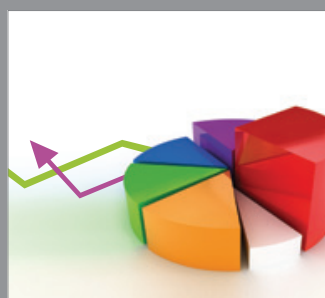

ournal of

Probability and Statistics

Promensencen
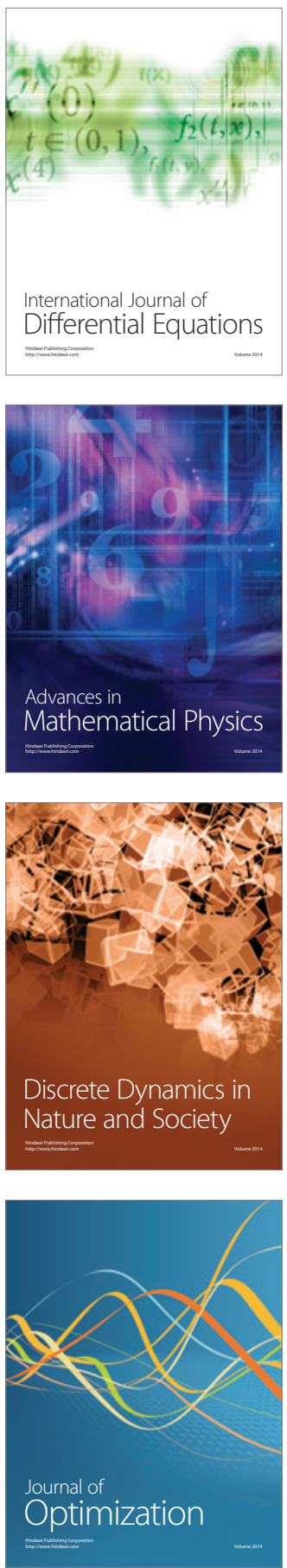